\title{
CINÉTICA DE FEIJÃO PRETO (PHASEOLUS VULGARIS, L.) EM SECADOR DE
} BANDEJA

\section{Deyzi Santos Gouveia}

Núcleo de Pesquisas Aplicadas em Ciência e Tecnologia de Alimentos, Instituto Federal de Educação Ciência e Tecnologia do Rio Grande do Norte, Campus Currais Novos, Mestre em Engenharia Agrícola; Professora Curso Técnico de Alimentos, Atuando na área de Análise físico-química e microbiológica de Alimentos. deyzigouveia@yahoo.com.br.

\section{Sânia Mara Pereira Ugulino}

Mestre em Engenharia Agrícola; graduada em Engenharia de Alimentos (UFPB)

Maria Elita Martins Duarte

Professor Associado da Universidade Federal de Campina Grande, lotada no Departamento de

Engenharia Agrícola; Doutora em Engenharia de Alimentos; atualmente exerce o cargo de coordenadora do curso de graduação em Engenharia de Alimentos.

Mario Eduardo Cavalcanti Mata

Professor Associado da Universidade Federal de Campina Grande, lotada no Departamento de

Engenharia Agrícola; Doutor em Engenharia de Alimentos

\section{RESUMO}

O presente trabalho propôs estudar a cinética de feijão preto (Phaseolus vulgaris, l.) em secador de bandeja a diferentes temperaturas do ar e posterior ajuste dos dados experimentais, obtidos com os diferentes modelos matemáticos ( Fick, Page e Cavalcanti Mata). O teor de umidade inicial das sementes foi determinado pelo método padrão da estufa, $105 \pm 3{ }^{\circ} \mathrm{C}$, durante 72 h, com três repetições, de acordo com as regras do Instituto Adolfo Lutz (1985). Os tratamentos de secagem foram realizados em cinco níveis de temperaturas $(40,50,-60,70$ e $80{ }^{\circ} \mathrm{C}$ ). Para cada tratamento de secagem foram utilizados em torno de $60 \mathrm{~g}$ de feijão por repetição. Para este fim utilizou-se um desidratador de frutas, Polidryer PD-25. O ar foi aquecido por meio de gás de cozinha, enquanto a temperatura foi controlada com o auxílio de um termopar, conectado ao secador. Os resultados mostraram que as equações propostas por Cavalcanti Mata e Page foram as que melhor representaram os dados experimentais, quando comparada com a equação de Fick utilizando um termo da série.

PALAVRAS-CHAVE: Cinética, feijão, secador

\section{KINETICS OF BLACK BEAN (Phaseolus vulgaris, L.) IN TRAY DRYER}

\begin{abstract}
This study proposed to study the kinetics of black bean (Phaseolus vulgaris L.) in tray dryer at different air temperatures and subsequent adjustment of experimental data with different mathematical models (Fick, Page Cavalcanti and Mata). the initial moisture content of seeds was determined by standard oven, $105 \pm 3{ }^{\circ} \mathrm{C}$ for $72 \mathrm{~h}$ with three replicates, according to the rules of the Institute Adolfo Lutz (1985). The drying treatments were carried out in five levels of temperatures $\left(40,50,60,70\right.$ and $\left.80{ }^{\circ} \mathrm{C}\right)$. For each drying treatment were used about 60 grams of beans per replicate. To this end we used a dehydrator fruit, polidryer pd-25. the air was heated with cooking gas, while the temperature was controlled with the aid of a thermocouple, connected to the dryer. The results showed that the equations proposed by
\end{abstract}


Cavalcanti Mata and Page were the ones that best represented the experimental data, when compared with the Fick equation using a term in the series.

KEY-WORDS: Kinetics, beans, dryer 


\section{CINÉTICA DE FEIJÃO PRETO (PHASEOLUS VULGARIS, L.) EM SECADOR DE} BANDEJA

\section{INTRODUÇÃO}

No Brasil, a produção de feijão cresceu de 2.006.005 t em 1987, com uma área colhida de 5.221.794 ha, para 2.822.340 t em 1996, com uma área colhida de 4.944.699 ha devido, principalmente, à adoção de um conjunto de tecnologias e insumos mais modernos. Com a expansão da cultura do feijão nas principais regiões produtoras do país, técnicas modernas de secagem vêm sendo desenvolvidas para atender às crescentes necessidades dos produtores, com o objetivo de simplificar e reduzir os custos associados ao processo produtivo (AFONSO JUNIOR, 1999).O feijão é cultivado e consumido mundialmente e é a leguminosa mais consumida na América Latina. $\mathrm{O}$ baixo valor biológico das proteínas do feijão pode ser resultante, dentre outros, da presença de fatores anti-nutricionais presentes no tegumento do grão (CHIARADIA, 1999).

O feijão tem importância econômica para o país, pois o Brasil é um dos maiores produtores e consumidores mundiais deste grão. Porém, as perdas pós-colheita, causadas por más condições de armazenamento, são responsáveis por quebras significativas na produção deste grão (DIAS, 2000). A demanda dos consumidores por alimentos mais frescos e convenientes tem aumentado muito nas últimas décadas. Uma das técnicas de preservação de alimento mais antigas utilizadas pelo homem consiste na remoção de umidade dos alimentos pelo processo de secagem. Em relação aos outros métodos de conservação para períodos longos como a refrigeração, a apertização ou tratamentos químicos, a desidratação oferece custo mais baixo e operações mais simples.

A remoção de umidade provoca a diminuição da atividade de água do produto, inibindo o desenvolvimento de microrganismos e retardando deteriorações de origem físico-química (CHAUCA, 2004).A secagem de grãos é uma prática importante, utilizada principalmente com o objetivo de aumentar o tempo de conservação dos grãos, levando assim a um melhor aproveitamento da produção agrícola. Tradicionalmente a secagem ao sol tem sido usada para secar a maioria das sementes de leguminosas maduras, porém, o uso de equipamentos de secagem, leva a produtos de qualidade mais elevada. De acordo com FIOREZE (2004), produtos biológicos com umidade inicial acima de 70 a $75 \%$, base úmida, no início do processo de secagem apresentam a superfície coberta por uma película de água, e nesta situação a taxa de secagem (perda de umidade em função do tempo) é função de três parâmetros externos do ar de secagem: velocidade, temperatura e umidade. Se as condições do ar de secagem são fixas, a taxa de secagem será constante. Este fenômeno é observado também em produtos nos quais a resistência interna à migração de umidade é menor que a resistência externa para evaporar e remover o vapor de água da superfície do produto.

A secagem é uma prática das mais antigas de conservação de alimentos desenvolvida pelo homem. Alimentos de origem vegetal como cereais feijão e ervilhas, quando colhidos suficientemente secos e adequadamente armazenados, permanecem em condições de consumo e/ ou industrialização por longos períodos de tempo (AFONSO JÚNIOR et al., 1999). Existem hoje muitos tipos de secadores que podem ser utilizados na desidratação de alimentos, porém, a escolha de um determinado secador depende da natureza da matériaprima, do produto final a ser obtido, dos aspectos econômicos e das condições de operação. De um modo geral, os secadores podem ser divididos em duas categorias distintas. Os secadores adiabáticos e os secadores por contato. Os secadores adiabáticos são aqueles que se utilizam do ar para fazer a transferência de calor necessária. São eles: secadores de cabine ou armário, secadores de túnel, secador por aspersão ou atomizador (spray-drying) e fornos 
secadores. Nos secadores por contato o calor é transportado por condução ao alimento através de uma superfície metálica, estática ou em movimento, são eles: Secador de tambor e desidratadores a vácuo.

\section{CINÉTICA DE SECAGEM}

A obtenção da cinética de secagem é de fundamental importância para a modelagem matemática da operação e projeto de secadores. É através deste estudo que se estabelecem as equações da umidade em função do tempo de secagem para os diferentes períodos de taxas de secagem. A cinética de secagem possibilita também a determinação do mecanismo predominante na transferência de massa do material para o fluido e as respectivas equações matemáticas correspondentes (FERREIRA,2004). O estudo de sistemas de secagem, seu dimensionamento, otimização e a determinação da viabilidade de sua aplicação comercial, podem ser feitos por simulação matemática. Para a simulação, cujo princípio se fundamenta na secagem de sucessivas camadas do produto, utiliza-se um modelo matemático que representa satisfatoriamente a perda de umidade do produto durante o período de secagem. $\mathrm{Na}$ literatura, encontram-se vários métodos propostos para se analisar a secagem em camada fina de produtos higroscópicos: os teóricos, os semi-teóricos e os empíricos.

\section{SECAGEM EM CAMADA FINA}

De acordo com FIOREZE (2004), em certos processos de secagem, o produto é inteiramente envolvido pelo ar de secagem em regime turbulento, como por exemplo, em secagem tipo leito fluidizado, secador de tambor rotativo, secagem de camadas finas, etc. Nesses processos, teoricamente, toda a superfície de uma partícula isolada é área de troca de calor e massa, não havendo contato físico entre as partículas suficiente para troca de calor por condução, ou troca de massa por difusão entre elas. O estudo em camada fina é o mais empregado para a determinação da cinética de secagem sendo que, a temperatura, umidade relativa e velocidade permanecem constantes, ou seja, as condições do fluido de secagem podem ser consideradas não alteradas quando em contato com o sólido. Assim, é possível a determinação de parâmetros de secagem e equações para a taxa de secagem (FERREIRA,2004). Os resultados obtidos através de estudo em camada fina são indispensáveis na predição das equações de taxa de secagem utilizadas na modelagem dos fenômenos de transferência de calor e massa em leitos de camada espessa (MASSARANI et al. , 1992).

Assim, o presente trabalho teve como objetivo determinar, experimentalmente, as curvas de secagem para sementes de feijão e ajustar diferentes modelos matemáticos aos dados experimentais, em função da temperatura do ar de secagem e do teor de umidade inicial do produto.

\section{MATERIAL E MÉTODOS}

No Laboratório de Processamento e Armazenamento de Produtos Agrícolas do Departamento de Engenharia Agrícola do Centro de Ciência e Tecnologia da Universidade Federal de Campina Grande foi realizada a prática de secagem. Foram utilizadas feijão preto (Phaseolus vulgaris ), comprado em supermercado. $\mathrm{O}$ teor de umidade inicial das sementes foi determinado pelo método padrão da estufa, $105 \pm 3{ }^{\circ} \mathrm{C}$, durante $72 \mathrm{~h}$, com três repetições, de acordo com as regras do Instituto Adolfo Lutz (1985). Os tratamentos de secagem foram realizados em cinco níveis de temperaturas $\left(40,50,60,70\right.$ e $\left.80{ }^{\circ} \mathrm{C}\right)$. Para cada tratamento de secagem foram utilizados em torno de $60 \mathrm{~g}$ de feijão por repetição. Para este fim utilizou-se um desidratador de frutas, Polidryer PD-25 (Figura 1). 


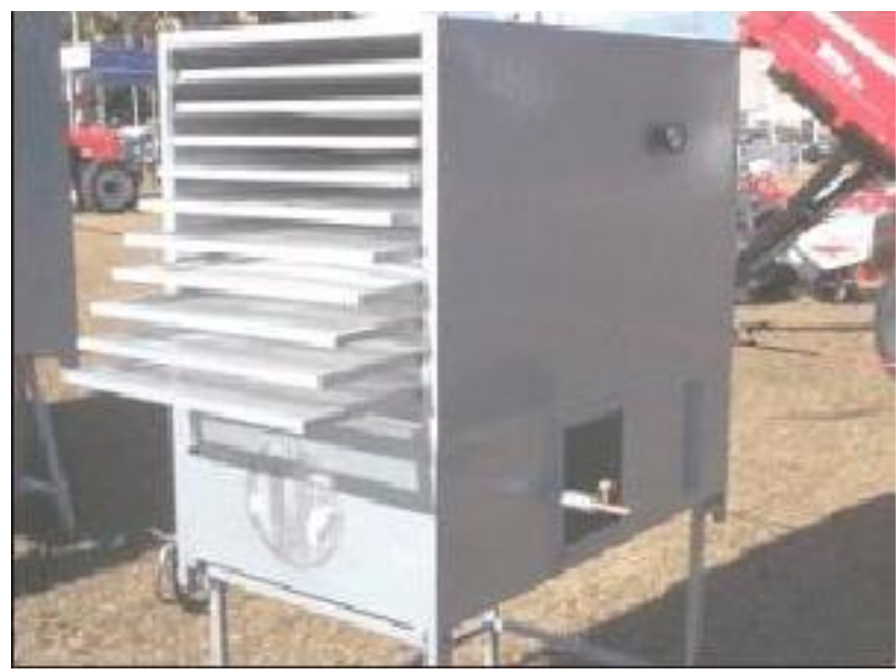

Figura 1 - Secador de bandejas Polidryer PD-25, para desidratação de frutas

$\mathrm{O}$ ar foi aquecido por meio de gás de cozinha, enquanto a temperatura foi controlada com o auxílio de um termopar, conectado ao secador.

Para determinação do teor de água inicial foi utilizada a fórmula:

$$
X b u(\%)=\frac{P i-P f}{P i}
$$

onde:

Pi: peso inicial, em gramas;

Pf: peso final, em gramas.

Utilizou-se a fórmula 2 para acompanhar a umidade do produto no processo de secagem, através da variação de peso como parâmetro de determinação:

$$
X b u=100-\left[\frac{P_{i}\left(100-X_{i}\right)}{P_{f}}\right]
$$

onde:

Xbu: teor de água em base úmida;

$\mathrm{P}_{\mathrm{i}}$ : peso inicial do produto a ser seco

$\mathrm{P}_{\mathrm{f}}$ : peso final do produto após secagem;

$\mathrm{X}_{1}$ : umidade inicial do produto.

Para a determinação do teor de água em base seca foi utilizada a seguinte fórmula:

$$
X b s=\frac{X b u}{(1-X b u)}
$$


onde:

Xbs: teor de água em base seca

Xbu: teor de água em base úmida

Para determinação da Razão de Umidade foi utilizada a seguinte fórmula:

$$
R X=\frac{X b s-X e}{X b s_{\text {inicial }}-X e}
$$

onde:

RX: razão de umidade (adimensional)

Xe: teor de água de equilíbrio em base seca

Xbs: teor de água em base seca

Xbs (inicial): Umidade inicial em base seca

Obs.: Substituir em todas as umidades o $\underline{\mathbf{U}}$ por $\underline{\mathbf{X}}$.

Tabela 1. Modelos matemáticos de regressão não-linear para predizer a secagem do feijão preto (Phaseolus vulgaris, $L$.)

\begin{tabular}{|c|c|c|}
\hline Item & Equação & Autores \\
\hline 01 & $R X=\frac{X-X_{e}}{X_{i}-X_{e}}=\exp \left(-k t^{n}\right)$ & PAGE \\
\hline 02 & $R X=\frac{X-X_{e}}{X_{i}-X_{e}}=a_{1} \exp \left(-a_{2} t^{a_{3}}\right)+a_{4} \exp \left(-a_{2} t^{a_{5}}\right)+a_{6}$ & CAVALCANTI MATA \\
\hline 03 & $R X=\frac{6}{\pi^{2}} \cdot \exp \left[\frac{\pi^{2}}{R^{2}} \cdot D \cdot t\right.$ & FICK com um termo da série \\
\hline
\end{tabular}

Sendo:

RX = Razão de umidade;

$\mathrm{X}=$ teor de água inicial;

$\mathrm{X}_{\mathrm{e}}=$ teor de água de equilíbrio;

$\mathrm{X}_{\mathrm{i}}=$ teor de água no instante;

$\mathrm{k}, \mathrm{n}, a_{1}, a_{2}, a_{3}, a_{4}, a_{5}$ e $a_{6}=$ são parâmetros das equações.

\section{RESULTADOS E DISCUSSÃO}

Nas Tabelas 2, 3 e 4, estão apresentados os resumos dos modelos ajustados por regressão não linear aos dados experimentais da secagem do feijão preto em camada fina, considerando-se a 
temperatura do ar de secagem e o teor de umidade inicial do produto como variáveis independentes.

\section{EQUAÇÃO DE PAGE}

Na Figura 2 estão expressos os dados experimentais e calculados com a equação de Page, nas secagens feitas às temperaturas de $40,50,60,70$ e $80^{\circ} \mathrm{C}$ para o feijão preto.

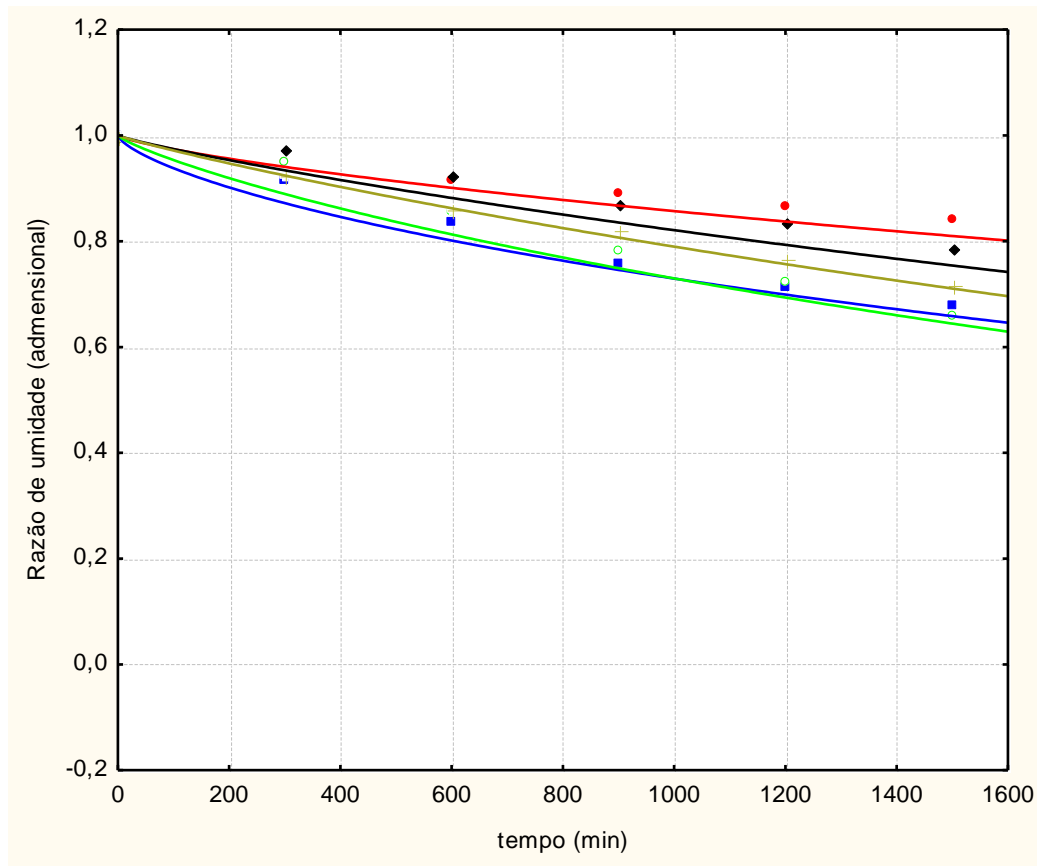

- Dados experimentais da secagem a $40^{\circ} \mathrm{C}$ $R U=\exp \left(-(0,763 e-3) \cdot t^{(0,768611)}\right)$

- Dados experimentais da secagem a $50^{\circ} \mathrm{C}$ RU $=\exp \left(-(0,002618) \cdot \mathrm{t}^{(0,693623)}\right)$

- Dados experimentais da secagem a $60^{\circ} \mathrm{C}$ $R U=\exp \left(-(0,444 \mathrm{e}-3) \cdot \mathrm{t}^{(0,882542)}\right)$

Dados experimentais da secagem a $70^{\circ} \mathrm{C}$ $\mathrm{RU}=\exp \left(-(0,001082) \cdot \mathrm{t}^{(0,821365)}\right)$

Dados experimentais da secagem a $80^{\circ} \mathrm{C}$ $\mathrm{RU}=\exp \left(-(0,431 \mathrm{e}-3) \cdot \mathrm{t}^{(0,912747)}\right)$

Figura 2 - Curvas de secagem dos dados experimentais e calculados do feijão preto pela equação de Page, obtidas por regressão não linear às temperaturas de 40, 50, 60,70 e $8^{\circ} \mathrm{C}$

Os coeficientes da equação de Page encontram-se na Tabela 2. Pode-se perceber que todas as curvas apresentam coeficientes de determinação superiores a 99,6\%, ou seja, os resultados indicam um bom ajuste da equação proposta por Page aos dados experimentais. 
Tabela 2. Coeficientes da Equação de Page, obtidas por regressão não linear em secagem do feijão preto às temperaturas de $40,50,60,70 \mathrm{e} 80^{\circ} \mathrm{C}$.

\begin{tabular}{cccc}
\hline \multicolumn{4}{c}{$\begin{array}{c}\text { Equação de Page } \\
\text { Ru=exp }\left(-\mathbf{k . t} \mathbf{t}^{\mathbf{n}}\right)\end{array}$} \\
$\mathrm{T}\left({ }^{\circ} \mathrm{C}\right)$ & $\mathrm{K}$ & $\mathrm{n}$ & $\mathrm{R}^{2}(\%)$ \\
40 & 0,000763 & 0,768611 & 0,99667 \\
50 & 0,002618 & 0,693623 & 0,99861 \\
60 & 0,000444 & 0,882542 & 0,99667 \\
70 & 0,001082 & 0,821265 & 0,99754 \\
80 & 0,000431 & 0,912747 & 0,99900 \\
\hline
\end{tabular}

\section{EQUAÇÃO DE CAVALCANTI MATA}

Na Figura 3 estão expressos os dados experimentais e calculados com a equação de Cavalcanti Mata nas secagens feitas às temperaturas de $40,50,60,70$ e $80^{\circ} \mathrm{C}$ para o feijão preto. Na Tabela 3 observa-se uma média de 99,79\% para os coeficientes de determinação obtendo, assim, um ótimo ajuste dessa equação aos dados experimentais da secagem.

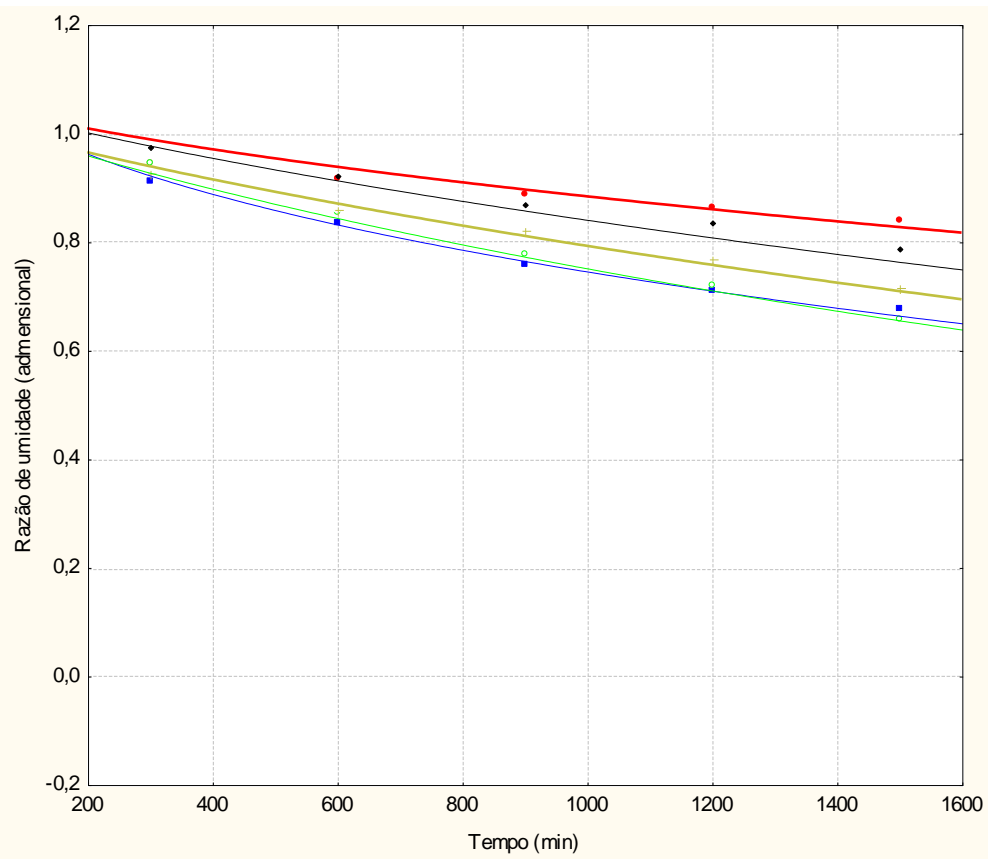

- Dados experimentais da secagem a $40^{\circ} \mathrm{C}$

$\mathrm{RU}=(, 712626) \cdot \exp \left(-(, 001154) \cdot \mathrm{t}^{(0,767172)}\right)+(, 494091) \cdot \exp \left(-(0,001154) \cdot \mathrm{t}^{(0,620654)}\right)+(-0,136664)$

- Dados experimentais da secagem a $50^{\circ} \mathrm{C}$

_ $R U=(-0,11688) \cdot \exp \left(-(0,005519) \cdot \mathrm{x}^{(2,25155)}\right)+(1,10781) \cdot \exp \left(-(0,005519) \cdot \mathrm{t}^{(0,622756)}\right)+(0,009077)$

- Dados experimentais da secagem a $60^{\circ} \mathrm{C}$ $R U=(0,961977) \cdot \exp \left(-\left(0,651 \mathrm{e}^{-3}\right) \cdot \mathrm{t}^{(0,862656)}\right)+(, 150954) \cdot \exp \left(-(0,651 \mathrm{e}-3) \cdot \mathrm{t}^{(0,648109)}\right)+(-0,04886)$

Dados experimentais da secagem a $70^{\circ} \mathrm{C}$ $R U=(0,863746) \cdot \exp \left(-\left(0,745 \mathrm{e}^{-3}\right) \cdot \mathrm{x}^{(0,897203)}\right)+(0,267588) \exp \left(-\left(0,745 \mathrm{e}^{-3}\right) \cdot \mathrm{x}^{(0,688929)}\right)+(-0,09236)$

Dados experimentais da secagem a $80^{\circ} \mathrm{C}$ $R U=(1,0756) \cdot \exp \left(-\left(0,662 \mathrm{e}^{-3}\right) \cdot \mathrm{t}^{(0,864978)}\right)+(-0,60738) \cdot \exp \left(-\left(0,662 \mathrm{e}^{-3}\right) \cdot \mathrm{t}^{(0,495364)}\right)+(0,558354)$

Figura 3 - Curvas de secagem dos dados experimentais e calculados do feijão preto pela

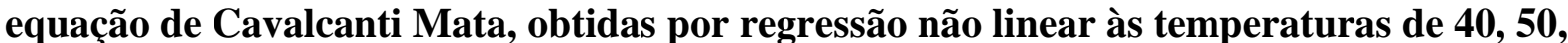
60,70 e $80^{\circ} \mathrm{C}$. 
Tabela 3 - Coeficientes da Equação de Cavalcanti Mata, obtidas por regressão não linear em secagem do feijão preto às temperaturas de $40,50,60,70$ e $80^{\circ} \mathrm{C}$

\section{Equação de Cavalcanti Mata}

\begin{tabular}{cccccccc}
\multicolumn{7}{c}{$R U=a_{1} \exp \left(-a_{2} t^{a_{3}}\right)+a_{4} \exp \left(-a_{2} t^{a_{5}}\right)+a_{6}$} \\
$\mathrm{~T}\left({ }^{\circ} \mathrm{C}\right)$ & $\mathrm{a}_{1}$ & $\mathrm{~K}_{1}$ & $\mathrm{a}_{2}$ & $\mathrm{a}_{3}$ & $\mathrm{a}_{4}$ & $\mathrm{a}_{5}$ & $\mathrm{R}^{2}(\%)$ \\
40 & 0,477563 & 0,001004 & 0,704125 & 0,516625 & 0,751000 & $-0,026344$ & 0,99309 \\
50 & $-0,116884$ & $-0,005519$ & 2,251547 & 1,107808 & 0,622756 & 0,009077 & 0,99961 \\
60 & 0,961977 & 0,000651 & 0,862656 & 0,150954 & 0,648109 & $-0,048861$ & 0,99874 \\
70 & 0,863746 & 0,000745 & 0,897203 & 0,267588 & 0,688929 & $-0,092357$ & 0,99935 \\
80 & 1,075599 & 0,000662 & 0,864978 & $-0,607383$ & 0,495364 & 0,558354 & 0,99917 \\
\hline
\end{tabular}

\section{EQUAÇÃO DE FICK}

Na Figura 4 estão expressos os dados experimentais e calculados com a equação de Fick nas secagens feitas às temperaturas de $40,50,60,70$ e $80^{\circ} \mathrm{C}$ para o feijão preto. Pela Tabela 4 observam-se os baixos valores dos coeficientes de determinação da equação de Fick com um termo da série tendo em média 89,93\%, apresentando assim os menores coeficientes com relação às outras equações estudadas.

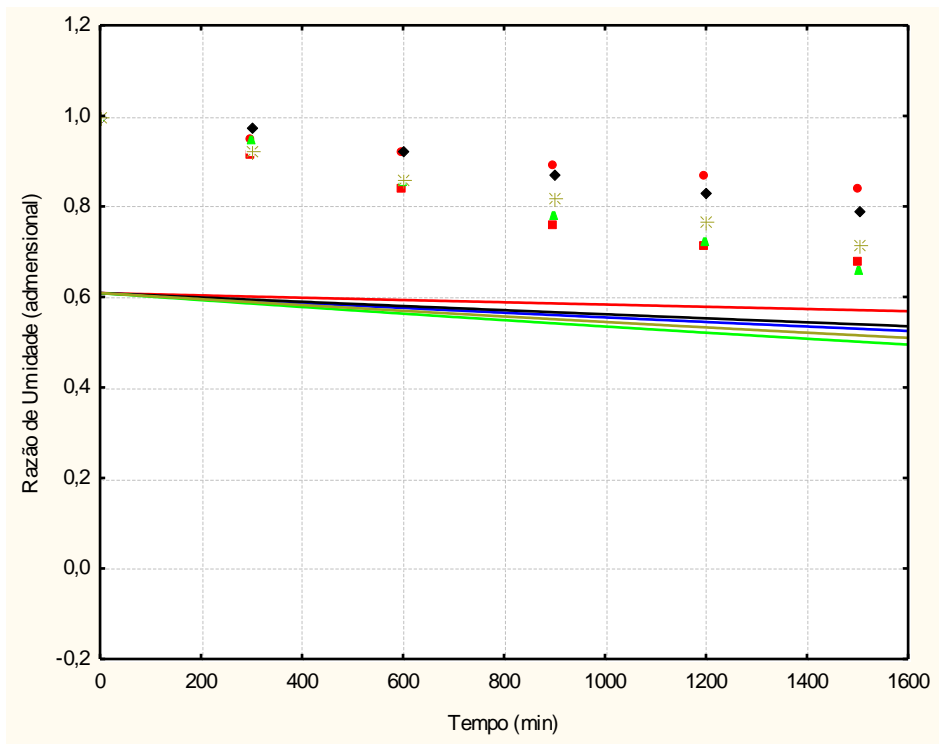

\footnotetext{
- Dados experimentais da secagem a $40^{\circ} \mathrm{C}$

$\mathrm{RU}=0,6085 \cdot \exp \left(\left((9,86 / 0,004)^{2}\right) \cdot\left(-0,7 \mathrm{e}-{ }^{11}\right) \mathrm{t}\right)$

- Dados experimentais da secagem a $50^{\circ} \mathrm{C}$ $R U=0,6085 \cdot \exp \left(\left(9,86 /(0,004)^{2}\right) \cdot\left(-0,15 \mathrm{e}^{-9}\right) \mathrm{t}\right)$

- Dados experimentais da secagem a $60^{\circ} \mathrm{C}$ $R U=0,6085 \cdot \exp \left(\left(9,86 /(0,004)^{2}\right) \cdot\left(-0,13 \mathrm{e}^{-9}\right) \mathrm{t}\right)$

Dados experimentais da secagem a $70^{\circ} \mathrm{C}$ $R U=0,6085 \cdot \exp \left(\left(9,86 /(0,004)^{2}\right) \cdot\left(-0,21 \mathrm{e}^{-9}\right) \cdot \mathrm{t}\right)$

3ados experimentais da secagem a $80^{\circ} \mathrm{C}$ $R U=0,6085 \cdot \exp \left(\left(9,86 /(0,004)^{2}\right) \cdot\left(-0,18 \mathrm{e}^{-9}\right) \cdot \mathrm{t}\right)$
}

Figura 4 - Curvas de secagem dos dados experimentais e calculados do feijão preto pela equação de Fick, obtidas por regressão não linear às temperaturas de 40, 50, 60,70 e $\mathbf{8 0}^{\circ} \mathrm{C}$. 
Tabela 4 - Coeficientes da Equação de Fick, obtidas por regressão não linear em secagem do feijão preto às temperaturas de $40,50,60,70$ e $80^{\circ} \mathrm{C}$

\begin{tabular}{ccc}
\hline \multicolumn{3}{c}{ Equação de Fick } \\
& $R U=\frac{6}{\pi^{2}} \cdot \exp \left[\frac{\pi^{2}}{r_{e}{ }^{2}} \cdot D \cdot t\right]$ & $\mathbf{R}^{\mathbf{2}}(\boldsymbol{\%})$ \\
$\mathbf{T}\left({ }^{\mathbf{o}} \mathbf{C}\right)$ & $\mathbf{D}\left(\mathbf{m}^{2} / \mathbf{s}\right)$ & 0,87012 \\
40 & $-0,7 \times 10^{-11}$ & 0,93081 \\
50 & $0,15 \times 10^{-9}$ & 0,88711 \\
60 & $-0,13 \times 10^{-9}$ & 0,90954 \\
70 & $-0,21 \times 10^{-9}$ & 0,89904 \\
80 & $-0,18 \times 10^{-9}$ & \\
\hline
\end{tabular}

\section{CONCLUSÃO}

Diante dos resultados obtidos dos modelos ajustados por regressão não linear aos dados experimentais da secagem do feijão preto em camada fina conclui-se que:

As médias dos coeficientes de determinação obtidos por Cavalcanti Mata, Page e Fick são respectivamente $99,6 \%, 99,76 \%$ e $89,93 \%$,

$>\quad$ Nas curvas de secagem, obtidas pelos modelos das equações de Cavalcanti Mata e Page, os valores observados estão bem próximos dos valores preditos;

$>\quad$ As equações propostas por Cavalcanti Mata e Page foram as que melhor representaram os dados experimentais, quando comparada com a equação de Fick utilizando um termo da série.

\section{REFERÊNCIAS}

1. AFONSO JÚNIOR, P.C.; CORRÊA, P. C. Comparação de modelos matemáticos para descrição da cinética de secagem em camada fina de sementes de feijão Revista Brasileira de Engenharia Agrícola e Ambiental, v.3, n.3, p.349-353, 1999 Campina Grande, PB, DEAg/UFPB

2. CHAUCA M. C. ; RAMOS A. M ; STRINGHETA, P. C. ; MARQUES, J. A.;SILVA, P. I. Curvas De Secagem E Avaliação Da Atividade De Água Da Banana Passa. B.CEPPA, Curitiba, v. 22, n. 1, jan./jun. 2004 B.CEPPA, Curitiba, v. 22, n. 1, p. 121132, jan./jun. 2004

3. CHIARADIA, A. C. N.; COSTA, N. M. B. ; GOMES, J. C. Retirada do tegumento e da extração dos pigmentos na qualidade protéica do feijão-preto Rev. Nutr., Campinas, 12(2): 131-136, maio/ago., 1999

4. DIAS, Mariângela C. et al . Effect of drying in two-dimensional spouted bed drying on physical and technologycal properties of black beans ( Phaseolus vulgaris, L). Ciênc. Tecnol. Aliment., Campinas, v. 20, n. 3, 2000.

5. FERREIRA, M. M. P. Desenvolvimento caracterização e secagem de sementes com cobertura artificial. 235f. Dissertação (Mestrado em Engenharia Química) Universidade Federal de São Carlos: UFSCar: 2004.

6. FIOREZE, R. Princípios de secagem de produtos biológicos. João Pessoa: UFPB, 2004 
7. ISNTITUTO ADOLFO LUTZ, São Paulo, Normas analíticas do Instituto Adolfo Lutz. 3ed. São Paulo: Instituto Adolfo Lutz, 1985.v1v533p

8. MASSARANI, G. e TELLES, A. S., Aspecto da secagem do material sólido particulado. In: Freire, J. T.e Sartori, D.J.M. Tópicos especiais em secagem. São Carlos, v1, ed. UFSCar, p1-30/2002 